\title{
Identification of exosomal miRNA biomarkers for diagnosis of papillary thyroid cancer by small RNA sequencing
}

\author{
Daofeng Dai ${ }^{1, *}$, Yawen Tan ${ }^{2, *}$, Liangfeng Guo ${ }^{2}$, Aifa Tang ${ }^{1}$ and Yongsheng Zhao' \\ ${ }^{1}$ Health Science Center, The First Affiliated Hospital of Shenzhen University, Department of Nuclear Medicine, \\ Institute of Translational Medicine, and Guangdong Key Laboratory of Systems Biology and Synthetic Biology for \\ Urogenital Tumors, Shenzhen Second People's Hospital, Shenzhen, Guangdong, China and ²Department of Breast \\ and Thyroid Surgery, Shenzhen Second People's Hospital, Shenzhen, Guangdong, China \\ Correspondence \\ should be addressed \\ to A Tang or Y Zhao \\ Email \\ tangaifa2018@email.szu.edu. \\ cn or zhaoys69@gmail.com
}

\begin{abstract}
Context: Exosomal miRNAs are considered potential non-invasive biomarkers for thyroid cancer. However, the global exosomal miRNAs profile for papillary thyroid cancer (PTC) has not been revealed.

Objective: This study investigated the diagnostic value of plasma and serum exosomal miRNAs for PTC.

Methods: Plasma and serum samples were collected from ten patients with benign thyroid nodules and 17 with PTC for small RNA sequencing. Plasma samples were collected from two independent cohorts, including 119 patients with PTC, 51 healthy people and 82 patients with benign thyroid nodules, for validation by quantitative reverse-transcription polymerase chain reaction (RT-qPCR).

Results: Small RNA sequencing identified 41 putative exosomal miRNA biomarkers for PTC. Twelve miRNAs were selected for validation. miR-376a-3p, miR-4306, miR-4433a-5p, and miR-485-3p expression significantly increased in patients with PTC compared to that in healthy people and patients with benign thyroid nodules $\left(P^{<} 0.05\right)$. Moreover, miR-485-3p and miR-4433a-5p presented larger areas under the curve (AUCs). The high expression of exosomal miR485-3p correlated with tumor size greater than or equal to $1 \mathrm{~cm}$, advanced clinical stage, extrathyroidal extension, BRAF mutation, and lymph node metastasis. Besides, miR-485-3p exhibited the highest AUCs in diagnosing PTC patients with high-risk factors.

Conclusions: Plasma exosomal miR-485-3p and miR-4433a-5p might serve as biomarkers for PTC diagnosis. Plasma exosomal miR-485-3p could enable discrimination between high-risk and low-risk PTC.
\end{abstract}

\section{Introduction}

Thyroid cancer (TC) is a common endocrine carcinoma and its incidence has increased over the last few decades (1). Papillary TC (PTC) accounts for up to $80 \%$ of all TC cases; thus, it is responsible for the increase in TC incidence (2). Despite relatively good curability and a high survival rate, there are still $10 \%$ of patients with PTC that die from the disease (3). Thus, it is urgent to develop a biomarker to distinguish high-risk patients with PTC from low-risk patients, which can provide better treatment to high-risk patients and prevent overtreatment of patients with low-risk PTC. Thyroid biopsy, an invasive operation, is still the standard method to diagnose PTC and evaluate the prognosis (4). Therefore, non-invasive biomarkers are needed for diagnosis and prognosis of PTC. (c) 2020 European Society of Endocrinology Printed in Great Britain
Published by Bioscientifica Ltd. 
miRNAs - a group of small noncoding RNA molecules with a length of 21-23 nucleotides (nts) - inhibit gene expression by combining with the 3 '-untranslated region of respective mRNAs $(5,6)$. miRNAs bind with multiple genes simultaneously; hence, the number of the target genes can occasionally be around 100 (7). Recent studies have shown that miRNAs are dysregulated in human malignancies and play an important role in the development and progression of cancer $(8,9,10)$. Functional studies demonstrate that miRNAs affect PTC cell proliferation, migration, and invasion $(11,12,13)$. Genome-wide profiling shows that hundreds of miRNAs are deregulated in tissues of patients with PTC, and several of these miRNAs are related to prognosis and can serve as diagnostic markers $(14,15)$.

Exosomes are vesicles with a size of $30-150 \mathrm{~nm}$ in diameter, consisting of a lipid bilayer in which proteins, nucleic acids, and lipids are packaged. Exosomes are important for cells to communicate with neighboring cells or with distant cells (16). All exosomes possess surface molecules that help them to target the recipient cells. Once attached to the recipient cells, the exosomes fuse with the cells' membranes to release their cargoes into target cells, thereby changing the physiological state of the recipient cells.

Exosomal miRNAs in biofluids are more stable, compared to free miRNAs in circulation (17). Recent studies have reported that exosomal miRNAs serve as diagnostic and prognostic biomarkers in several kinds of human cancers, such as glioma, bladder cancer, and breast cancer $(18,19,20)$. There are studies reporting that the expression levels of exosomal miRNAs in plasma of patients with PTC were significantly changed, suggesting that exosomal miRNAs have great potential to be biomarkers for PTC (21). The global profiling of exosomal miRNAs from plasma or serum of patients with PTC has not been explored.

In this study, we used small RNA sequencing to globally profile exosomal miRNA expression of plasma and serum samples from patients with PTC, compared the expression profiles of plasma and serum exosomal miRNAs to obtain exosomal miRNAs with the potential to be biomarkers, and utilized quantitative reverse-transcription polymerase chain reaction (RT-qPCR) to validate the potential biomarkers. We then analyzed the diagnostic and prognostic values of the remarkably upregulated exosomal miRNAs in plasma and serum as a promising liquid biopsy tool for PTC.

\section{Patients and methods}

\section{Patients}

Samples were collected from three cohorts. The screening set included ten patients with benign thyroid nodules and 17 patients with PTC; the training set comprised 21 healthy people, 23 patients with benign thyroid nodules and 23 patients with PTC. In addition, 30 healthy people, 59 patients with benign thyroid nodules, and 96 patients with PTC were enrolled in the validation cohort. Patients included in this study underwent thyroidectomy between January 2017 and September 2019 at Shenzhen Second People's Hospital. Archival slides of patients were evaluated by two pathologists. The clinicopathological information was supplied in Table 1. All samples were collected upon obtaining the written informed consent from patients and the prior approval for this study was granted by the Medical Ethics Committee of Shenzhen Second People's Hospital as per the Declaration of Helsinki.

\section{Plasma and serum preparation}

Peripheral blood samples with a minimum of $10 \mathrm{~mL}$ were collected preoperatively for plasma or serum preparation. Plasma was prepared by drawing peripheral blood into EDTA tubes. The serum was prepared by blood clotting at room temperature for less than $2 \mathrm{~h}$. Then, the tubes were first centrifuged at $1600 \boldsymbol{g}$ for $10 \mathrm{~min}$ at $4^{\circ} \mathrm{C}$, then centrifuged at $3000 \mathrm{~g}$ for $10 \mathrm{~min}$ at $4^{\circ} \mathrm{C}$ for both plasma and serum preparation. The supernatant was stored at $-80^{\circ} \mathrm{C}$ for further analysis.

\section{Isolation of exosomes}

Exosomes were isolated with a combination of centrifugation and ultracentrifugation. The plasma or serum samples were first centrifuged at $17000 \boldsymbol{g}$ for 30 min at $4^{\circ} \mathrm{C}$ to remove cell debris. The supernatant was collected, and the exosomes were pelleted at $120000 \boldsymbol{g}$ for $2 \mathrm{~h}$ at $4^{\circ} \mathrm{C}$ with the Optima XPN-100 ultracentrifuge (Beckman Coulter). The pellets were washed with $11 \mathrm{~mL}$ of phosphate-buffered saline (PBS). The ultracentrifugation step was repeated. Finally, the exosomal pellet was diluted in de-ionized water.

\section{Nanoparticle tracking analysis}

Exosomes isolated from plasma or serum were diluted in $1 \mathrm{~mL}$ of $1 \times \mathrm{PBS}$, and nanoparticle tracking analysis 
Table 1 Clinical features of PTC patients in three cohorts.

\begin{tabular}{|c|c|c|c|}
\hline Clinical features & Screening set & Training set & Validation set \\
\hline Total number & 17 & 23 & 96 \\
\hline Age (years)* & $58.3(19-79)$ & $57.9(24-81)$ & $56.6(22-83)$ \\
\hline \multicolumn{4}{|l|}{ Tumor size $(\mathrm{cm})$} \\
\hline$\geq 1$ & 10 & 14 & 56 \\
\hline$<1$ & 7 & 9 & 40 \\
\hline \multicolumn{4}{|l|}{$\begin{array}{l}\text { Lymph node } \\
\text { metastasis }\end{array}$} \\
\hline NO & 8 & 11 & 44 \\
\hline N1 & 9 & 12 & 52 \\
\hline \multicolumn{4}{|l|}{$\begin{array}{c}\text { Extrathyroidal } \\
\text { extension }\end{array}$} \\
\hline Yes & 6 & 8 & 37 \\
\hline No & 11 & 15 & 59 \\
\hline \multicolumn{4}{|l|}{ Clinical stage } \\
\hline$|-| \mid$ & 12 & 14 & 63 \\
\hline III-IV & 5 & 9 & 33 \\
\hline \multicolumn{4}{|l|}{ BRAF mutation } \\
\hline Negative & 6 & 7 & 31 \\
\hline Positive & 11 & 16 & 65 \\
\hline
\end{tabular}

*Age at the time of specimen draw, median (range).

(NTA) for exosomes was performed with ZETASIZER Nano series-Nano-ZS (Malvern, UK) and its corresponding software. Exosomal particle concentrations and size distribution profiles were calculated.

\section{Transmission electron microscopy}

The exosomes isolated by ultracentrifugation were resuspended in PBS and applied to a carbon-coated copper grid for $20 \mathrm{~min}$. Excess liquid was blotted using filter paper. Then, $2 \%$ phosphotungstic acid solution (Sigma) was added to yield negative staining for $10 \mathrm{~min}$ at room temperature. After repeatedly removing the unnecessary liquid by filter paper, the copper grids were dried for several minutes using an incandescent lamp. The microphotographs were obtained using an HT7700 transmission electron microscope (Hitachi, Japan).

\section{Western blot analysis}

Exosomal protein was prepared with a radioimmunoprecipitation assay (RIPA) buffer supplemented with protease inhibitor cocktail (Abcam). Twenty micrograms of exosomal protein were separated on a $10 \%$ sodium dodecyl sulfate polyacrylamide gel electrophoresis (SDS-PAGE), and then transferred to a PVDF membrane (Millipore). Membranes were incubated with primary antibodies against CD63 (ProteinTech) or CD81 (Abcam) at a dilution of 1:1000. Membranes were incubated with goat anti-rabbit IgG secondary antibodies (ProteinTech) at a dilution of 1:3000.

\section{RNA extraction and RT-qPCR}

Total RNA was extracted from exosomal samples with TRIzol Reagent (Invitrogen) according to the manufacturer's instructions. The quantity and purity of the RNA were measured by NanoDrop 2000 (Thermo Fisher Scientific).

Due to the lack of a consensus housekeeping miRNA in plasma for RT-qPCR, the synthetic cel-miR-39 (Ribobio, China), used as the internal control for plasma samples, was spiked into each RNA sample prior to cDNA synthesis. miRNA expression was analyzed using MiR-X miRNA qRTPCR SYBR Kit (Takara) and miDETECT A Track ${ }^{\mathrm{TM}}$ miRNA RT-qPCR Primers (Ribobio). The quantitative PCR assays were performed on the QuantStudio DX Real-Time PCR System (Applied Biosystems). The relative amount of exosomal miRNAs was calculated using the equation $2^{-\Delta C t}$.

\section{Small RNA sequencing}

We used 0.2-1.0 $\mu \mathrm{g}$ of RNA sample to construct small RNA sequencing libraries. Following separation of small RNA by PAGE and addition of the 3 ' adaptors, RT primers and $5^{\prime}$ adaptors to the selected RNA, single-stranded cDNA was generated using Superscript II Reverse Transcriptase (Invitrogen). Several rounds of PCR amplification were performed to enrich the cDNA fragments. The PCR products of $100-120$ bp were purified via PAGE, followed by heat denaturation and circularization. The single-stranded circular DNA was used as the final library. Sequencing was performed on BGISEQ-500. The low-quality reads, adaptors and other contaminants were eliminated to get clean reads. Clean reads for each sample were aligned to the most recent mirBASE database. The small RNA expression level is calculated by using transcripts per kilobase per million mapped reads (TPM). $P$ value was adjusted as $P_{\text {adj }}$. A $P_{\text {adj }}$-value $<0.05$ was considered statistically significant.

\section{Statistical analysis}

Statistical analyses were performed using GraphPad Prism 5.0 software. The nonparametric Mann-Whitney test and Kruskal-Wallis test were used to determine statistically significant differences in miRNA expression among groups, with a $P$ value $<0.05$ as the significant 
level. Receiver-operating characteristic (ROC) curves were plotted using each miRNA expression value. The area under the curve (AUC) with 95\% CI was calculated for each ROC curve.

\section{Results}

\section{Isolation and characterization of plasma and serum exosomes}

To ensure the quality of exosomes from plasma or serum samples, the isolated exosomes were characterized by NTA, transmission electron microscopy (TEM), and Western blot. The NTA results showed that the exosomes were approximately $130 \mathrm{~nm}$ in diameter, and the exosomes from plasma and serum samples had a similar size distribution and peak region (Fig. 1A). The negativestaining TEM of plasma or serum exosomes also illustrated a typical diameter of 30-150 nm and bilayer membrane structure (Fig. 1B). Western blot was performed to ensure the expression of established biomarkers for exosomes. As shown in Fig. 1C, the plasma and serum exosomes both had markedly detectable CD63 and CD81.

\section{Identification of putative plasma and serum exosomal miRNA biomarkers for PTC}

Small RNA sequencing was first performed on exosomal miRNAs in plasma and serum samples from 17 patients

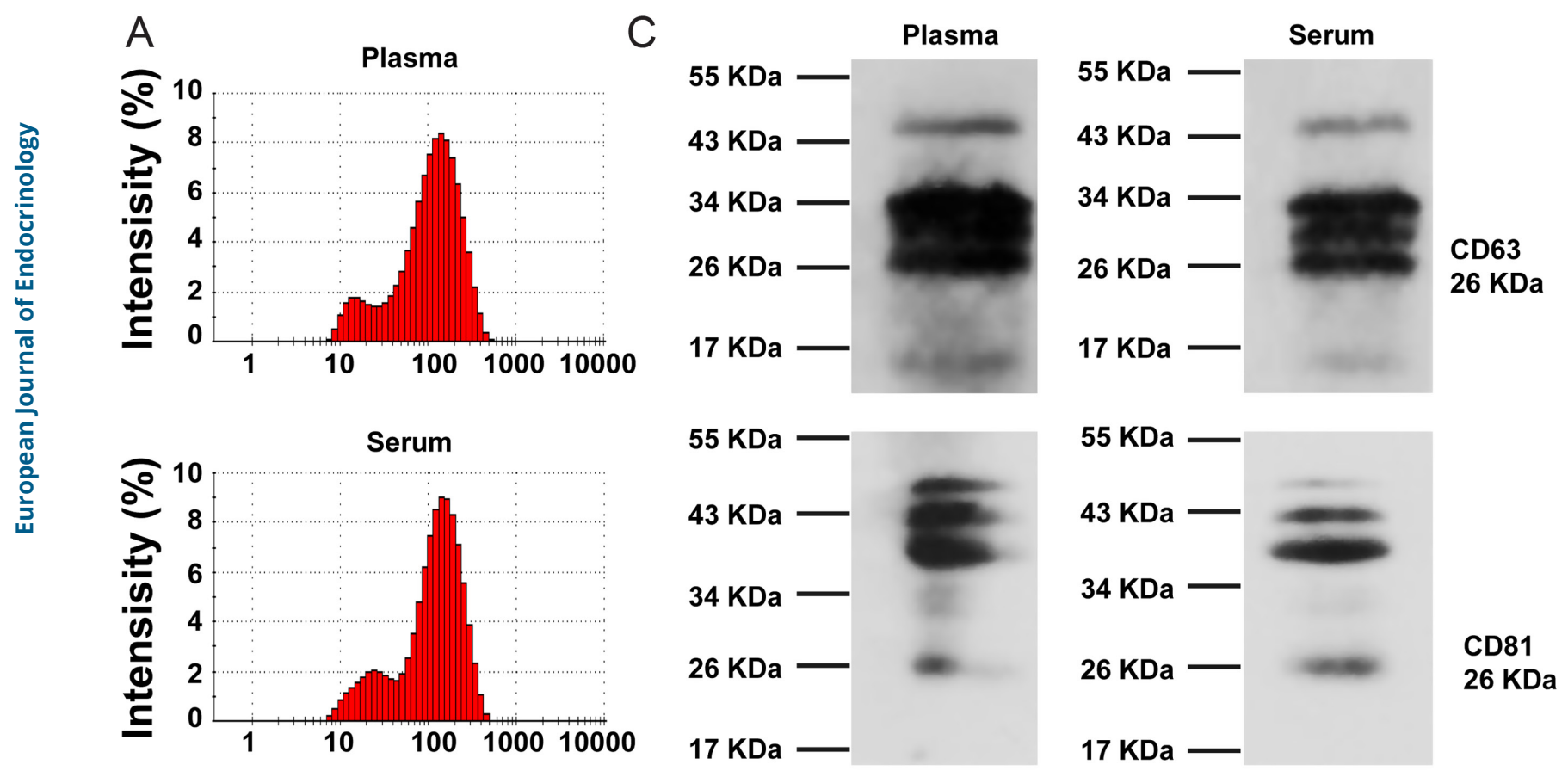

B

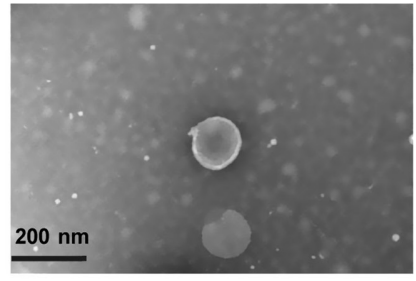

Plasma

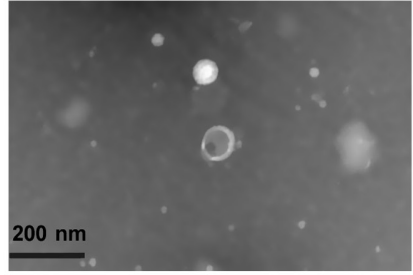

Serum

\section{Figure 1}

Characterization of exosomes from plasma or serum samples. (A) Size distribution and concentration of plasma or serum exosomes by nanoparticle tracking analysis. (B) Negative-staining transmission electron microscopy of plasma or serum exosomes. Solid black lines represent $200 \mathrm{~nm}$ scale bars. (C) Western blot analysis of plasma or serum exosomes with exosomal marker antibodies CD63 and CD81. 

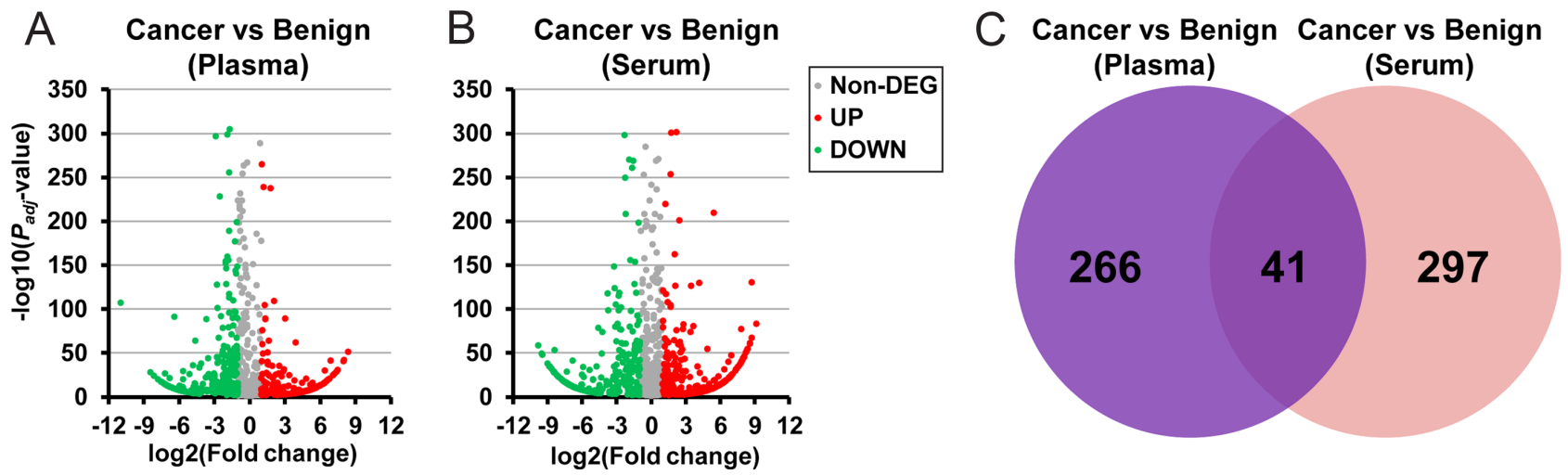

Figure 2

Differentially expressed exosomal miRNAs in patients with papillary thyroid cancer (PTC) were assessed by small RNA sequencing. (A) Volcano plot shows the distribution of differentially expressed exosomal miRNAs in plasma samples from patients with PTC $(n=17)$ as compared with patients with benign thyroid nodules $(n=10)$. (B) Expression profiles of exosomal miRNAs in serum samples from patients with PTC. (C) Venn diagram exhibits significantly upregulated miRNAs in plasma and serum exosomes from patients with PTC as compared with patients with benign thyroid nodules. $P_{\text {adj; }}$ is the adjusted $P$ value.

with PTC and 10 patients with benign thyroid nodules in the screening set. Comparing the small RNA sequencing data of plasma exosomes from patients with PTC and patients with benign thyroid nodules, we found 307 upregulated miRNAs and 342 downregulated miRNAs (Fig. 2A). The volcano plot in Fig. 2B showed significantly different profiles of serum exosomal miRNAs between patients with PTC and patients with benign thyroid nodules, which included 338 upregulated miRNAs and 269 downregulated miRNAs. Among the upregulated miRNAs, only 41 were concurrent both in plasma and serum exosomes (Fig. 2C). These overlapped miRNAs were considered putative biomarkers. However, the expression of many exosomal miRNAs was extremely low, which hampers their implementation as biomarkers due to undetectability. Only 12 miRNAs (expression value $>4$ both in plasma and serum samples from PTC patients) were selected for further validation (Table 2).

\section{Validation of plasma exosomal miRNAs as biomarkers for PTC}

To validate the exosomal miRNAs identified by small RNA sequencing, 12 miRNAs in plasma exosomes were analyzed by RT-qPCR in the training set. Five miRNAs, including miR-204-3p, miR-376a-3p, miR-4306, miR4433a-5p, and miR-485-3p, were significantly increased in patients with PTC $(n=23)$ as compared with patients with benign thyroid nodules $(n=23)(P<0.05$, Fig. 3A). To analyze the expression of these miRNAs in healthy people, the training set also included 21 healthy controls.
The expression levels of miR-204-3p, miR-376a-3p, miR-4306, miR-4433a-5p, and miR-485-3p were also significantly increased in patients with PTC as compared with that in healthy controls $(P<0.01$, Fig. 3A). However, there was no significant difference in the expression of these five miRNAs between patients with benign thyroid nodules and healthy controls. The five exosomal miRNAs were chosen for further validation in a larger cohort (validation set) involving 30 healthy controls, 59 patients with benign thyroid nodules, and 96 patients with PTC. Consistent with results of the training set, the expression of miR-376a-3p, miR-4306, miR-4433a-5p, and miR485-3p was significantly elevated in patients with PTC compared with that in healthy controls and patients with benign thyroid nodules $(P<0.01)$ (Fig. 3B). The expression of miR-204-3p was significantly increased in patients with PTC as compared with that in healthy controls $(P<0.01)$. However, miR-204-3p expression was not significantly elevated in patients with PTC as compared with that in patients with benign thyroid nodules.

\section{The diagnostic value of putative exosomal miRNA biomarkers}

Subsequently, ROC curve analysis was performed to investigate the diagnostic value of miR-204-3p, miR376a-3p, miR-4306, miR-4433a-5p, and miR-485-3p for PTC. The AUCs of the five miRNAs to discriminate patients with PTC from patients with benign thyroid nodules ranged from 0.5985 to 0.8581 (Fig. 4A, B, C, D and E). Among them, miR-485-3p presented the largest AUC 


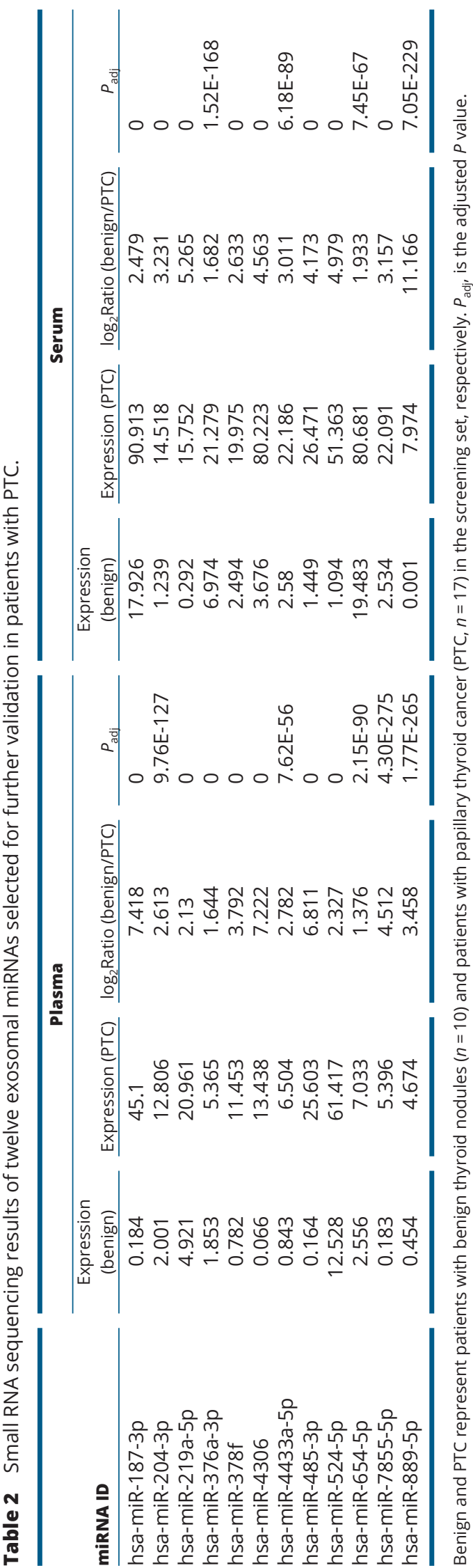

(0.8581; 95\% CI: 0.802-0.9141; sensitivity $=85.42 \%$, and specificity $=73.33 \%$ ) (Fig. 4E), followed by miR-4433a-5p (0.8122; 95\% CI: 0.7358-0.8886; sensitivity=83.33\%, and specificity $=73.33 \%$ ) (Fig. 4D). ROC curve analysis for miRNAs to distinguish patients with PTC from healthy controls showed that the AUCs for miR-485-3p and miR-4433a-5p were 0.866 (95\% CI: 0.795-0.9369; sensitivity $=80.21 \%$, and specificity $=71.19 \%$ ) and 0.8628 (95\% CI: $0.791-0.9347$; sensitivity $=81.25 \%$, and specificity $=72.88 \%$ ), respectively (Fig. 4D and E). These results suggested that exosomal miR-485-3p and miR4433a-5p have relatively high diagnostic accuracy in discriminating patients with PTC from healthy people and patients with benign thyroid nodules and can be the non-invasive biomarkers for PTC.

\section{The association between the expression of exosomal miRNAs and clinical features of PTC}

Evaluation of the relationship between the expression of the five exosomal miRNAs and the clinical parameters of PTC showed that miR-485-3p and miR-4433a-5p expression was associated with several clinicopathological features. The high expression of exosomal miR-485-3p was associated with tumor size greater than or equal to $1 \mathrm{~cm}(P<0.01)$, advanced clinical stage $(P<0.001)$, extrathyroidal extension $(P<0.001)$, BRAF mutation $(P<0.001)$, and lymph node metastasis $(P<0.001)$ (Fig. 5). The high expression of exosomal miR-4433a-5p was related with advanced clinical stage $(P<0.05)$, extrathyroidal extension $(P<0.01)$, BRAF mutation $(P<0.001)$, and lymph node metastasis $(P<0.001)$ (Fig. 5). High expression of miR-204-3p was associated with tumor size greater than or equal to $1 \mathrm{~cm}(P<0.001)$, while high expression of miR-4306 was associated with tumor size less than $1 \mathrm{~cm}(P<0.01)$ (Fig. 5A).

Next, ROC curve analysis was performed to evaluate the diagnostic accuracy of the five exosomal miRNAs in differentiating between PTC patients with different clinical characteristics in the validation set. Among the five miRNAs, miR-485-3p possessed the highest accuracy in discriminating PTC patients at the advanced stage (stage III-IV) from patients at the early stage (stage I-II) (AUC $=0.7532 ;$ 95\% CI: 0.6494-0.8571; Fig. 6B), PTC patients with extrathyroidal extension from patients without extrathyroidal extension $(\mathrm{AUC}=0.7265 ; 95 \% \mathrm{CI}$ : 0.6191-0.8339; Fig. 6C), PTC patients with BRAF mutation from patients without BRAF mutation $(\mathrm{AUC}=0.8903 ; 95 \%$ CI: 0.8255-0.9551; Fig. 6D), PTC patients with lymph node metastasis from patients without lymph node metastasis 
A miR-204-3p

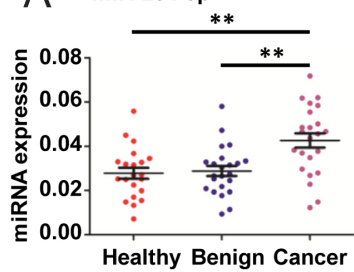

Healthy Benign Cancer

B miR-204-3p
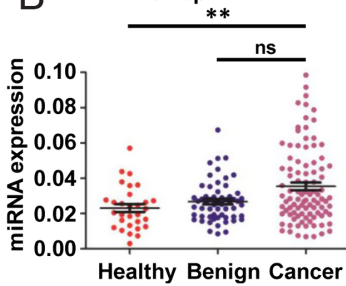

miR-376a-3p

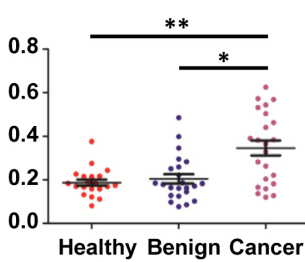

Healthy Benign Cancer

miR-376a-3p

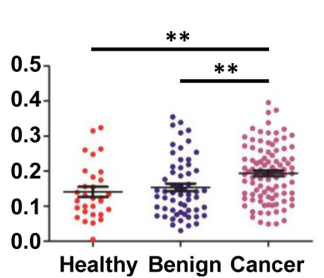

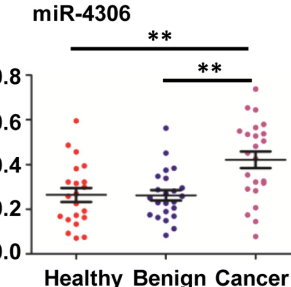

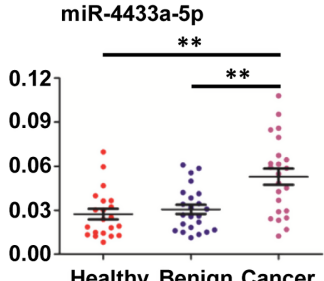

miR-4306

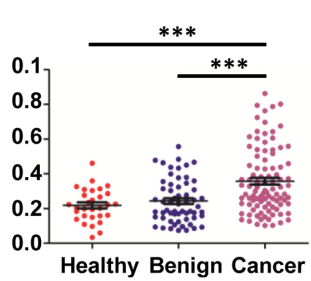

miR-4433a-5p

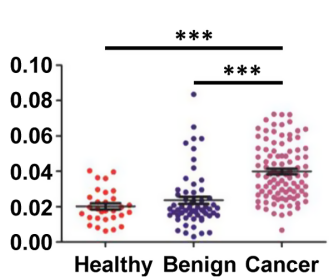

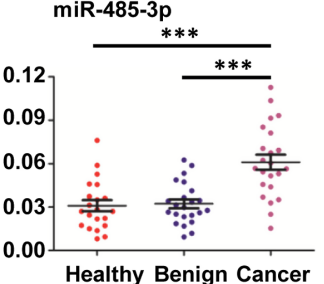

miR-485-3p

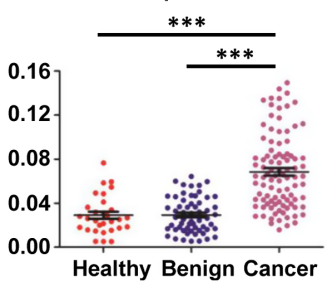

\section{Figure 3}

Quantitative reverse-transcription polymerase chain reaction (RT-qPCR) validation of candidate exosomal miRNAs. The expression of candidate exosomal miRNAs was validated in plasma samples from 21 and 30 healthy controls, 23 and 59 patients with benign thyroid nodules, and 23 and 96 patients with PTC in the training set (A) and the validation set (B), respectively. The data were normalized to cel-miR-39 as an internal control. Kruskal-Wallis tests were performed. $P_{\text {adj }}$ is the adjusted $P$ value. ${ }^{\star} P_{\text {adj }}<0.05$; $\star * P_{\text {adj }}<0.01 ; * \star * P_{\text {adj }}<0.001$.

$(\mathrm{AUC}=0.8051 ; 95 \%$ CI: 0.7175-0.8926; Fig. 6E). Exosomal miR-204-3p had the highest accuracy in differentiating PTC patients with tumor size $\geq 1 \mathrm{~cm}$ from patients with tumor size $<1 \mathrm{~cm}$ (AUC=0.7984; 95\% CI: 0.7122-0.8847; Fig. 6A). These results indicate that exosomal miR-485-3p can distinguish high-risk from low-risk PTC patients.

\section{Discussion}

Several promising forms of biomarkers for liquid biopsy are currently available. For instance, cell-free DNA detection has achieved great success in several types of cancers, such as non-small-cell lung cancer (22, 23).
However, this method is not suitable for PTC because the DNA mutation rate and the detection rate of cell-free DNA mutations are both extremely low in patients with PTC $(24,25)$. Further, RNA-type molecules in biofluids are unstable as they are readily hydrolyzed by nuclease, which also curbs the application of cell-free RNA molecules as biomarkers. miRNAs in exosomes are very stable because exosomes have membranes that shelter miRNAs from hydrolysis by nuclease (17). Exosomal miRNA profiles have been examined in several types of carcinomas (18, $19,20)$. However, to date, only one study assessing the exosomal miRNAs of patients with PTC that reported the differentially expressed exosomal miRNAs in 50 patients with PTC and follicular TC by RT-qPCR (21). However, the
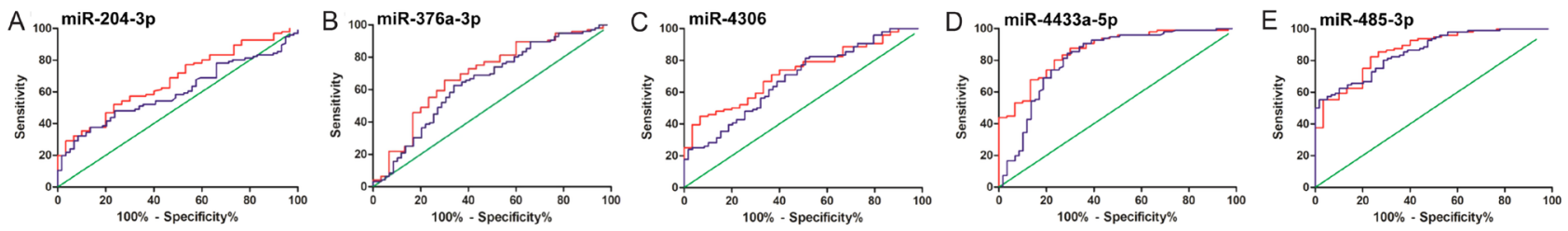

$$
- \text { Cancer vs Healthy } \quad-\text { Cancer vs Benign }
$$

\section{Figure 4}

Receiver-operating characteristic (ROC) curves for five selected exosomal miRNAs. (A, B, C, D and E) ROC curves for five selected exosomal miRNAs were plotted based on quantitative reverse-transcription polymerase chain reaction (RT-qPCR) results. The ROC curves to discriminate patients with PTC from healthy controls in the validation set are marked by red lines; the ROC curves to differentiate patients with PTC from patients with benign thyroid nodules in the validation set are marked by blue lines. 

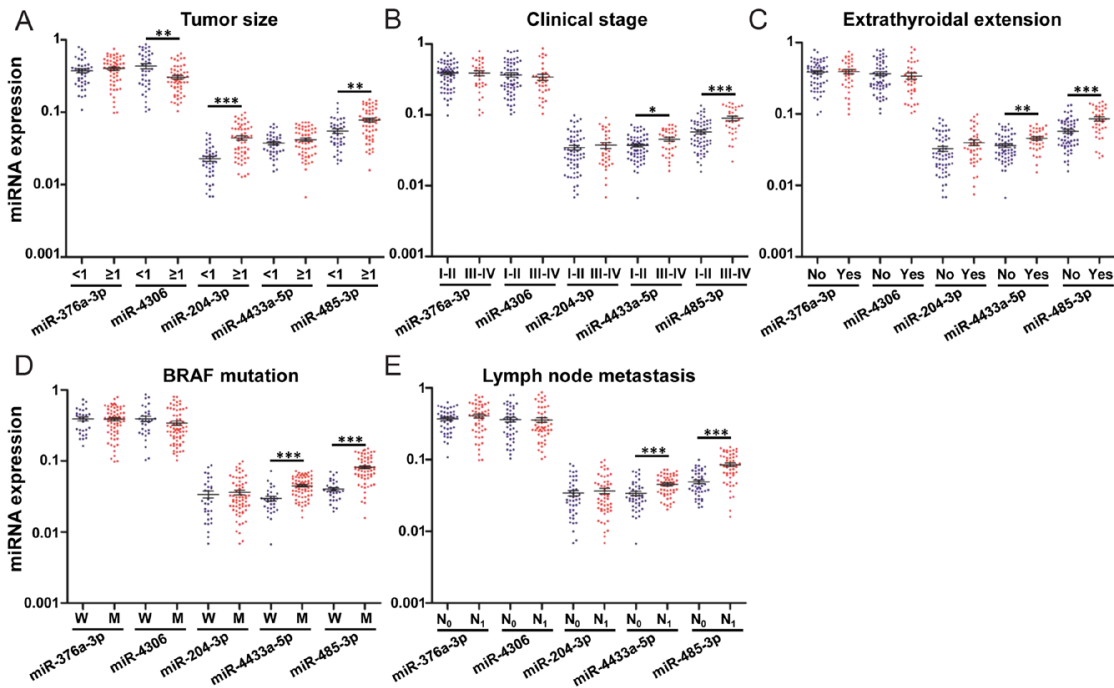

\section{Figure 5}

The association between expression levels of candidate exosomal miRNAs and clinical features in the validation set. (A) The expression of five candidate exosomal miRNAs in PTC patients of different tumor sizes. (B) The expression of five candidate exosomal miRNAs in PTC patients with different clinical stage. (C) The expression of five candidate exosomal miRNAs in PTC patients with or without extrathyroidal extension. (D) The expression of five candidate exosomal miRNAs in PTC patients with or without BRAF mutation. ' $W$ ' represents PTC patients without BRAF mutation; ' $M$ ' represents PTC patients with BRAF mutation. (E) The expression of five candidate exosomal miRNAs in PTC patients with or without lymph node metastasis. $* P<0.05$; $* * P<0.01$; $\star * \star P<0.001$. study above implemented a small sample size, and the global signature of exosomal miRNAs for patients with PTC has not been thoroughly examined.

In this study, we investigated the exosomal miRNA profiles of patients with PTC, using a large sample size with small RNA sequencing combined with RT-qPCR validation. We identified 41 significantly upregulated miRNAs concurrently expressed in plasma and serum exosomes from patients with PTC, as compared with those from patients with benign thyroid nodules. Among them, four exosomal miRNAs, including miR-376a-3p, miR4306, miR-4433a-5p, and miR-485-3p, were determined to be significantly and steadily increased in plasma samples from patients with PTC in comparison with those from healthy controls and patients with benign thyroid nodules by RT-qPCR in two independent cohorts. miR-485-3p and miR-4433a-5p held relatively high diagnostic accuracy in sorting patients with PTC from healthy controls and patients with benign thyroid nodules. These results indicate plasma exosomal miR-485-3p and miR-4433a-5p have considerable potential as diagnostic biomarkers for PTC. Moreover, the high expression of exosomal miR485-3p correlated with tumor size greater than or equal to $1 \mathrm{~cm}$, advanced clinical stage, extrathyroidal extension,
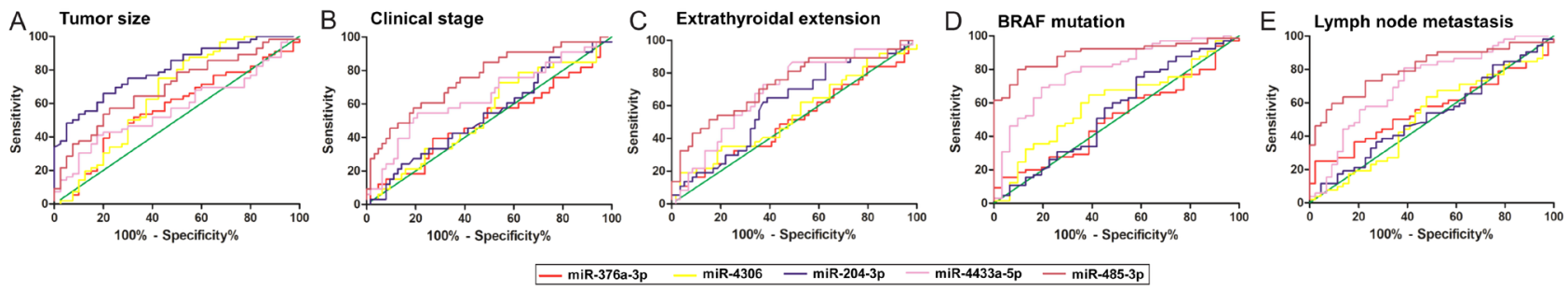

miR-4306 $\quad-$ miR-204-3p

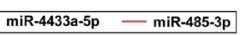

\section{Figure 6}

Comparison of receiver-operating characteristic (ROC) curves for five selected exosomal miRNAs to distinguish between PTC patients with different clinical features in the validation set. ROC curve analysis for the 5 exosomal miRNAs to discriminate PTC patients with tumor size $\geq 1$ from patients with tumor size $<1$ (A), PTC patients with stage III-IV from patients with stage I-II (B), PTC patients with extrathyroidal extension from patients without extrathyroidal extension (C), PTC patients with BRAF mutation from patients without BRAF mutation (D), PTC patients with lymph node metastasis from patients without lymph node metastasis (E). 
BRAF mutation, and lymph node metastasis. miR485-3p exhibited the highest accuracy in discriminating PTC patients at the advanced stage from patients at the early stage, patients with extrathyroidal extension from patients without extrathyroidal extension, patients with BRAF mutation from patients without BRAF mutation, patients with lymph node metastasis from patients without lymph node metastasis. These results suggest that the high expression of plasma exosomal miR-485-3p is related to poorer prognoses. Therefore, exosomal miR485-3p may serve as a promising biomarker to tell apart high-risk and low-risk PTC.

Furthermore, we identified and confirmed exosomal miR-204-3p as a biomarker for PTC (tumor size $\geq 1 \mathrm{~cm}$ ) diagnosis, and exosomal miR-4306 as a biomarker for PTC (tumor size $<1 \mathrm{~cm}$ ) diagnosis.

miR-485 showed a significantly lower expression in PTC tissues as compared with normal tissues (26). As the targets of miR-485, 47 mRNAs were associated with several biological processes, especially pathways in cancer. Among these mRNAs, STAT3 and SP1 have been experimentally validated as a tumor promoter in PTC $(27,28)$. Reportedly, miR-485-3p is a tumor suppressor in breast cancer (29). Low serum level of miR-485-3p could predict poor survival in patients with glioblastoma (30). Our data showed that miR-485-3p expression was markedly increased in plasma exosomes from patients with PTC as compared with healthy controls and patients with benign thyroid nodules, and the high expression of exosomal miR-485-3p was associated with high-risk factors, indicating that exosomal miR-485-3p may play a key role in progression of PTC.

A recent study showed that miR-204-3p was related to PTC progression, by acting as the target of lncRNA LINC00514 (31). miR-204-3p has been reported to be specifically detected in exosomes from the cerebrospinal fluid of healthy human donors (32). However, we found that miR-204-3p was also detected in plasma or serum exosomes in this study. Saiselet et al. reported that miR204-3p was downregulated in PTC tissues as compared to those in normal tissues (33). Notably, miRNA levels in exosomes do not necessarily reflect that in tissues, since the miRNA sorting mechanism may affect the incorporation of miRNA into exosomes. It was shown that miR-4306 served as a biomarker to predict survival and disease control of patients with head and neck cancer negative for human papillomavirus infection (34). miR-4306 was shown to be significantly upregulated in pancreatic cancer serum exosomes (35). Thus, the exosomal miRNAs identified in this study likely play essential roles in PTC. miR-4433a-5p was found to be significantly downregulated in peripheral blood from patients with vitiligo, which was not further validated (36). Because miR-4433a-5p was a newly identified miRNA, no study has reported the existence of miR-4433a-5p in exosomes. miR-204-3p, miR-4306, miR-4433a-5p, and miR-485-3p have never been found in plasma or serum exosomes from patients with PTC. Thus, this study shows that small RNA sequencing is a valid method to identify novel exosomal miRNA-based biomarkers for PTC.

Our study had several advantages. First, instead of measuring several miRNAs selected from different literature, we screened the genome-wide exosomal miRNA profiles of PTC via small RNA sequencing. Small RNA sequencing can measure the absolute abundance of miRNAs, enhancing the chance to identify differentially and steadily expressed exosomal miRNA. Furthermore, we performed small RNA sequencing both in plasma and serum samples and screened the differentially expressed exosomal miRNAs that co-occurred in those samples. This approach enabled us to eliminate many miRNAs that were significantly increased due to technical and human errors that might have occurred during experimental handling. Besides, we validated the expression of putative exosomal miRNAs biomarkers in two independent and large cohorts.

In summary, we performed a detailed analysis of the plasma and serum exosomal miRNA profile in patients with PTC, and we validated plasma exosomal miR-485-3p and miR-4433a-5p as non-invasive biomarkers differentiate patients with PTC from healthy people and patients with benign thyroid nodules. Moreover, plasma exosomal miR485-3p could serve as a biomarker to distinguish high-risk from low-risk PTC. These results suggest that miR-485-3p plays a pivotal role in the pathogenesis and development of PTC, and the functional role of exosomal miR-485-3p is an exciting subject requiring further study.

\section{Declaration of interest}

The authors declare that there is no conflict of interest that could be perceived as prejudicing the impartiality of this study.

\section{Funding}

This work was supported by China Postdoctoral Science Foundation (2018M633215), Shenzhen Science and Technology Innovation Commission (20140414170821273), Shenzhen Health and Family Planning Commission (KQTD20170810160226082, JCYJ20140414170821273), Shenzhen Health and Family Planning Commission (201401024), and State Engineering Laboratory for Tumor (High-Level Hospital Fund). 


\section{References}

1 Siegel RL, Miller KD \& Jemal A. Cancer statistics, 2018. CA: A Cancer Journal for Clinicians 201868 7-30. (https://doi.org/10.3322/ caac. 21442)

2 Pellegriti G, Frasca F, Regalbuto C, Squatrito S \& Vigneri R. Worldwide increasing incidence of thyroid cancer: update on epidemiology and risk factors. Journal of Cancer Epidemiology 2013 2013 965212. (https://doi.org/10.1155/2013/965212)

3 Hughes DT, Haymart MR, Miller BS, Gauger PG \& Doherty GM. The most commonly occurring papillary thyroid cancer in the United States is now a microcarcinoma in a patient older than 45 years. Thyroid 201121 231-236. (https://doi.org/10.1089/thy.2010.0137)

4 Gharib H, Papini E, Paschke R, Duick DS, Valcavi R, Hegedus L, Vitti P \& American Association of Clinical Endocrinologists. Associazione Medici Endocrinologi, and Europeanthyroid Association medical guidelines for clinical practice for the diagnosis and management of thyroid nodules. Endocrine Practice 201016 (Supplement 1) 1-43. (https://doi.org/10.4158/10024.GL)

5 Szeto CC. Urine miRNA in nephrotic syndrome. Clinica Chimica Acta 2014436 308-313. (https://doi.org/10.1016/j.cca.2014.06.016)

6 Mitchell PS, Parkin RK, Kroh EM, Fritz BR, Wyman SK, PogosovaAgadjanyan EL, Peterson A, Noteboom J, O'Briant KC, Allen A et al. Circulating microRNAs as stable blood-based markers for cancer detection. PNAS 2008105 10513-10518. (https://doi.org/10.1073/ pnas.0804549105)

7 Heneghan HM, Miller N \& Kerin MJ. MiRNAs as biomarkers and therapeutic targets in cancer. Current Opinion in Pharmacology 2010 10 543-550. (https://doi.org/10.1016/j.coph.2010.05.010)

8 Svoboda M, Izakovicova Holla L, Sefr R, Vrtkova I, Kocakova I, Tichy B \& Dvorak J. Micro-RNAs miR125b and miR137 are frequently upregulated in response to capecitabine chemoradiotherapy of rectal cancer. International Journal of Oncology 200833 541-547. (https:// doi.org/10.3892/ijo_00000038)

9 Orellana EA \& Kasinski AL. MicroRNAs in cancer: a historical perspective on the path from discovery to therapy. Cancers 20157 1388-1405. (https://doi.org/10.3390/cancers7030842)

10 Cuellar TL \& McManus MT. MicroRNAs and endocrine biology. Journal of Endocrinology 2005187 327-332. (https://doi.org/10.1677/ joe.1.06426)

11 Wang XZ, Hang YK, Liu JB, Hou YQ, Wang N \& Wang MJ. Overexpression of microRNA-375 inhibits papillary thyroid carcinoma cell proliferation and induces cell apoptosis by targeting ERBB2. Journal of Pharmacological Sciences 2016130 78-84. (https://doi. org/10.1016/j.jphs.2015.12.001)

12 Rossi ED, Bizzarro T, Martini M, Capodimonti S, Sarti D, Cenci T, Bilotta M, Fadda G \& Larocca LM. The evaluation of miRNAs on thyroid FNAC: the promising role of miR-375 in follicular neoplasms. Endocrine 201654 723-732. (https://doi.org/10.1007/s12020-0160866-0)

13 Lima CR, Geraldo MV, Fuziwara CS, Kimura ET \& Santos MF. MiRNA146b-5p upregulates migration and invasion of different papillary thyroid carcinoma cells. BMC Cancer 201616 108. (https://doi. org/10.1186/s12885-016-2146-z)

14 Swierniak M, Wojcicka A, Czetwertynska M, Stachlewska E, Maciag M, Wiechno W, Gornicka B, Bogdanska M, Koperski L, de la Chapelle A et al. In-depth characterization of the microRNA transcriptome in normal thyroid and papillary thyroid carcinoma. Journal of Clinical Endocrinology and Metabolism 201398 E1401-E1409. (https://doi.org/10.1210/jc.2013-1214)

15 Peng Y, Li C, Luo DC, Ding JW, Zhang W \& Pan G. Expression profile and clinical significance of microRNAs in papillary thyroid carcinoma. Molecules 201419 11586-11599. (https://doi. org/10.3390/molecules190811586)

16 Colombo M, Raposo G \& Thery C. Biogenesis, secretion, and intercellular interactions of exosomes and other extracellular vesicles.
Annual Review of Cell and Developmental Biology 201430 255-289. (https://doi.org/10.1146/annurev-cellbio-101512-122326)

17 Cheng L, Sun X, Scicluna BJ, Coleman BM \& Hill AF. Characterization and deep sequencing analysis of exosomal and non-exosomal miRNA in human urine. Kidney International 201486 433-444. (https://doi.org/10.1038/ki.2013.502)

18 Armstrong DA, Green BB, Seigne JD, Schned AR \& Marsit CJ. MicroRNA molecular profiling from matched tumor and bio-fluids in bladder cancer. Molecular Cancer 201514 194. (https://doi. org/10.1186/s12943-015-0466-2)

19 Shao N, Xue L, Wang R, Luo K, Zhi F \& Lan Q. miR-454-3p is an exosomal biomarker and functions as a tumor suppressor in glioma. Molecular Cancer Therapeutics 201918 459-469. (https://doi. org/10.1158/1535-7163.MCT-18-0725)

20 Hannafon BN, Trigoso YD, Calloway CL, Zhao YD, Lum DH, Welm AL, Zhao ZJ, Blick KE, Dooley WC \& Ding WQ. Plasma exosome microRNAs are indicative of breast cancer. Breast Cancer Research 201618 90. (https://doi.org/10.1186/s13058-016-0753-x)

21 Samsonov R, Burdakov V, Shtam T, Radzhabova Z, Vasilyev D, Tsyrlina E, Titov S, Ivanov M, Berstein L, Filatov M et al. Plasma exosomal miR-21 and miR-181a differentiates follicular from papillary thyroid cancer. Tumour Biology 201637 12011-12021. (http s://doi.org/10.1007/s13277-016-5065-3)

22 Douillard JY, Ostoros G, Cobo M, Ciuleanu T, McCormack R, Webster A \& Milenkova T. First-line gefitinib in Caucasian EGFR mutation-positive NSCLC patients: a phase-IV, open-label, singlearm study. British Journal of Cancer 2014110 55-62. (https://doi. org/10.1038/bjc.2013.721)

23 Douillard JY, Ostoros G, Cobo M, Ciuleanu T, Cole R, McWalter G, Walker J, Dearden S, Webster A, Milenkova T et al. Gefitinib treatment in EGFR mutated Caucasian NSCLC: circulating-free tumor DNA as a surrogate for determination of EGFR status. Journal of Thoracic Oncology 20149 1345-1353. (https://doi.org/10.1097/ JTO.0000000000000263)

24 Cancer Genome Atlas Research Network. Integrated genomic characterization of papillary thyroid carcinoma. Cell 2014159 676-690. (https://doi.org/10.1016/j.cell.2014.09.050)

25 Kim BH, Kim IJ, Lee BJ, Lee JC, Kim IS, Kim SJ, Kim WJ, Jeon YK, Kim SS \& Kim YK. Detection of plasma BRAF(V600E) mutation is associated with lung metastasis in papillary thyroid carcinomas. Yonsei Medical Journal 201556 634-640. (https://doi.org/10.3349/ ymj.2015.56.3.634)

26 Jiang W, Zhan H, Jiao Y, Li S \& Gao W. A novel lncRNA-miRNAmRNA network analysis identified the hub lncRNA RP11-159F24.1 in the pathogenesis of papillary thyroid cancer. Cancer Medicine 20187 6290-6298. (https://doi.org/10.1002/cam4.1900)

27 Mo XM, Li L, Zhu P, Dai YJ, Zhao TT, Liao LY, Chen GG \& Liu ZM. Up-regulation of Hsp27 by ER $\alpha / \mathrm{Sp} 1$ facilitates proliferation and confers resistance to apoptosis in human papillary thyroid cancer cells. Molecular and Cellular Endocrinology 2016431 71-87. (https:// doi.org/10.1016/j.mce.2016.05.010)

28 Xu Y, Han YF, Zhu SJ, Dong JD \& Ye B. miRNA-148a inhibits cell growth of papillary thyroid cancer through STAT3 and PI3K/AKT signaling pathways. Oncology Reports 201738 3085-3093. (https:// doi.org/10.3892/or.2017.5947)

29 Lou C, Xiao M, Cheng S, Lu X, Jia S, Ren Y \& Li Z. MiR-485-3p and miR-485-5p suppress breast cancer cell metastasis by inhibiting PGC-1 $\alpha$ expression. Cell Death and Disease 20167 e2159. (https://doi. org/10.1038/cddis.2016.27)

30 Wang ZQ, Zhang MY, Deng ML, Weng NQ, Wang HY \& Wu SX. Low serum level of miR-485-3p predicts poor survival in patients with glioblastoma. PLoS ONE 201712 e0184969. (https://doi.org/10.1371/ journal.pone.0184969)

31 Li X, Zhong W, Xu Y, Yu B \& Liu H. Silencing of lncRNA LINC00514 inhibits the malignant behaviors of papillary thyroid cancer through miR-204-3p/CDC23 axis. Biochemical and Biophysical Research 
Communications 2019508 1145-1148. (https://doi.org/10.1016/j. bbrc.2018.12.051)

32 Yagi Y, Ohkubo T, Kawaji H, Machida A, Miyata H, Goda S, Roy S, Hayashizaki Y, Suzuki H \& Yokota T. Next-generation sequencingbased small RNA profiling of cerebrospinal fluid exosomes. Neuroscience Letters 2017636 48-57. (https://doi.org/10.1016/j. neulet.2016.10.042)

33 Saiselet M, Gacquer D, Spinette A, Craciun L, Decaussin-Petrucci M, Andry G, Detours V \& Maenhaut C. New global analysis of the microRNA transcriptome of primary tumors and lymph node metastases of papillary thyroid cancer. BMC Genomics 201516828. (https://doi.org/10.1186/s12864-015-2082-3)

34 Hess J, Unger K, Maihoefer C, Schuttrumpf L, Wintergerst L, Heider T, Weber P, Marschner S, Braselmann H, Samaga D et al. A
five-microRNA signature predicts survival and disease control of patients with head and neck cancer negative for HPV infection. Clinical Cancer Research 201925 1505-1516. (https://doi. org/10.1158/1078-0432.CCR-18-0776)

35 Machida T, Tomofuji T, Maruyama T, Yoneda T, Ekuni D, Azuma T, Miyai H, Mizuno H, Kato H, Tsutsumi K et al. miR1246 and miR4644 in salivary exosome as potential biomarkers for pancreatobiliary tract cancer. Oncology Reports 201636 2375-2381. (https://doi. org/10.3892/or.2016.5021)

36 Shang Z \& Li H. Altered expression of four miRNA (miR-1238-3p, miR-202-3p, miR-630 and miR-766-3p) and their potential targets in peripheral blood from vitiligo patients. Journal of Dermatology 201744 1138-1144. (https://doi.org/10.1111/ 1346-8138.13886)

Received 9 July 2019

Revised version received 28 October 2019

Accepted 12 November 2019 